Extensive changes in innate immune gene expression in obese Göttingen minipigs do not lead to changes in concentrations of circulating cytokines and acute phase proteins

Højbøge, Tina Rødgaard; Skovgaard, K.; Moesgaard, Sophia Gry; Cirera Salicio, Susanna; Christoffersen, B. Ø.; Heegaard, Peter M. H.

Published in:

Animal Genetics

DOI:

10.1111/age. 12090

Publication date:

2013

Document version

Early version, also known as pre-print

Citation for published version (APA):

Højbøge, T. R., Skovgaard, K., Moesgaard, S. G., Cirera Salicio, S., Christoffersen, B. Ø., \& Heegaard, P. M. H. (2013). Extensive changes in innate immune gene expression in obese Göttingen minipigs do not lead to changes in concentrations of circulating cytokines and acute phase proteins. Animal Genetics, 45(1), 67-73. https://doi.org/10.1111/age.12090 


\title{
Extensive changes in innate immune gene expression in obese Göttingen minipigs do not lead to changes in concentrations of circulating cytokines and acute phase proteins
}

\author{
T. Rødgaard*, K. Skovgaard*, S. G. Moesgaard ${ }^{\dagger}, 1$, S. Cirera ${ }^{\dagger}$, B. $\varnothing$. Christoffersen $^{\ddagger}$ and \\ P. M. H. Heegaard* \\ *National Veterinary Institute, Technical University of Denmark, Frederiksberg C, 1870, Denmark. 'Department of Veterinary Clinical and \\ Animal Sciences, University of Copenhagen, Frederiksberg C, 1870, Denmark. "Novo Nordisk A/S, Maaløv, 2760, Denmark.
}

\begin{abstract}
Summary
The usefulness of Göttingen minipigs as models for obesity and obesity-related pathologies is well established. The low-grade inflammation associated with obesity involves a range of innate immune factors; however, to our knowledge, the impact of obesity on innate immune factor expression has not been studied in Göttingen minipigs. Therefore, we studied the expression of innate immune genes in liver and adipose tissues as well as serum concentrations of cytokines and acute phase proteins in obese vs. lean Göttingen minipigs. In the liver, of 35 investigated genes, the expression of nine was significantly different in obese pigs (three up-regulated, six down-regulated). Of 33 genes in adipose tissues, obesity was associated with changed expression of 12 genes in the visceral adipose tissue (VAT) (three up-regulated), 11 in the abdominal retroperitoneal adipose tissue (RPAT) (seven of these up-regulated) and eight in the subcutaneous adipose tissue (SAT) from the neck (five of which were up-regulated). Obesity-associated expression changes were observed for three genes in all adipose tissues, namely chemokine (C-C motif) ligand 3-like 1 (up-regulated), CD200 molecule (down-regulated) and interleukin 1 receptor antagonist (up-regulated) with interleukin 1 receptor antagonist being the most highly regulated gene in both VAT and RPAT. Looking at patterns of expression across the three types of adipose tissues, obesity was associated with an increased number of acute phase proteins differentially expressed between adipose tissues and a decreased tissue-specific expression of cytokines and chemokines. In contrast to obese humans, no changes in serum concentrations of haptoglobin, C-reactive protein, serum amyloid A, tumor necrosis factor- $\alpha$ and interleukin 6 were found in obese Göttingen minipigs.
\end{abstract}

Keywords comparative immunology, immune responses, inflammation, mRNA, obesity, pig model, porcine models

\section{Introduction}

Obesity has become an important health problem worldwide and is associated with elevated risks of several lifethreatening conditions, including cardiovascular diseases and type II diabetes (Nguyen \& Lau 2012). Obesity is

Address for correspondence

Professor Peter M. H. Heegaard, Innate Immunology Group, National Veterinary Institute, Technical University of Denmark, Bülowsvej 27, 1870 Frederiksberg C, Denmark.

E-mail: pmhh@vet.dtu.dk

${ }^{1}$ Present address: Novo Nordisk A/S Maaløv 2760 Denmark

Accepted for publication 19 July 2013 associated with chronic low-grade activation of the innate immune system, resulting in a chronic inflammation of the adipose tissue, which is thought to lead to the development of these disease states (Bastard et al. 2006; Gil et al. 2007; Federico et al. 2010). A wide range of factors of the innate immune response have been investigated with regard to obesity [reviewed, for example in the study by Federico et al. (2010) and Gil et al. (2007)]. The pig has been proposed as an excellent biomedical model for obesity as it is very similar to man with regard to anatomy, physiology, biochemistry and metabolism (Litten-Brown et al. 2010), and the use of the Göttingen minipig as a human obesity (Johansen et al. 2001) and diabetes (Larsen et al. 2002, 2007) model has been demonstrated. To further elucidate the usefulness of 
the Göttingen minipig as a human obesity model, it is important to include other molecular changes associated with obesity. The objective of this study was therefore to elucidate the effects of diet-induced obesity on the gene expression of innate immune factors in the liver and in three types of adipose tissues in the Göttingen minipig and to relate this to human obesity.

\section{Materials and methods}

\section{Animals, diets and sampling}

The study was approved by the Danish Animal Experiments Inspectorate. Fourteen female Göttingen minipigs (seven lean and seven obese) originating from Ellegaard Göttingen Minipigs A/S were included in the study. With the exception of one pair of half siblings (same mother) split between the two groups and one pair of half siblings (same father) in the obese group, all pigs in the two groups were unrelated at the parent level. The pigs were ovariectomized at 9-15 months of age to avoid hormonal effects on feed intake, and they were housed at the University of Copenhagen (Taastrup, Denmark) from 1-2 years of age and forward. The obese pigs had previously been used in pharmacological studies with therapeutic peptides, but had been subjected to a suitable washout period, based on the knowledge of the half-life of the peptides, prior to this study. Lean pigs were fed minipig standard chow Altromin $90232 \times 150 \mathrm{~g}$ a day and obese pigs had ad libitum access to minipig standard chow Altromin 9033 from the point of ovariectomization. The minipigs were euthanized with pentobarbital and exsanguinated at 41-47 months of age. Tissues were collected from the liver, subcutaneous adipose tissue (SAT) from the neck, mesenteric adipose tissue (VAT) and abdominal cranioventral retroperitoneal adipose tissue (RPAT) and snap-frozen in liquid nitrogen, and blood was collected for serum preparation. Tissue and serum samples were kept at $-80^{\circ} \mathrm{C}$ until analysis.

\section{Extraction of RNA}

All samples were homogenized on a gentle MACS Dissociator (Miltenyi Biotec) in gentleMACS M tubes (Miltenyi Biotec). For liver samples, RNA was extracted using the RNeasy Lipid Tissue Midi kit (Qiagen) according to manufacturer's protocol. All samples were subjected to on-column RNase-free DNase digestion (Qiagen).

For the adipose tissues, an optimized procedure was developed as follows: RNA was extracted with phenol and chloroform: Approximately $1 / 2$ gram of adipose tissue was homogenized with $4 \mathrm{ml}$ of QIAzol Lysis Reagent (Qiagen) on the gentleMACS Dissociator and then placed at room temperature for $5 \mathrm{~min}$. The homogenate was centrifuged at $12000 \boldsymbol{g}$ for $10 \mathrm{~min}$ at $4{ }^{\circ} \mathrm{C}$, after which the fatty upper layer was removed. Chloroform (Merck) was mixed with the remaining supernatant and placed at room temperature for 3 min after vigorous shaking for $15 \mathrm{~s}$. One $\mathrm{ml}$ of isopropanol (2-propanol; Merck) was added to the upper, aqueous phase and was centrifuged at $12000 \mathrm{~g}$ for $10 \mathrm{~min}$ at $4{ }^{\circ} \mathrm{C}$ after $5 \mathrm{~min}$ at room temperature. The pellet was washed 2 times in cold $75 \%$ ethanol, with a centrifugation step of 8 minutes at $8000 \mathrm{~g}$ at $4{ }^{\circ} \mathrm{C}$ between each wash and then dried at $55^{\circ} \mathrm{C}$ for $2 \mathrm{~min}$. The RNA was dissolved in RNase-free water at $55^{\circ} \mathrm{C}$ for $10 \mathrm{~min}$.

For all samples, the RNA yield was measured on the NanoDrop ND-1000 Spectrophotometer (NanoDrop Technologies, Inc.), and RNA integrity was measured with the Agilent RNA 6000 Nano Kit (Agilent Technologies) on the Agilent 2100 Bioanalyzer (Agilent Technologies) according to the manufacturer's protocol. Liver samples yielded RNA with an RNA integrity number (RIN) of $9.0 \pm 0.1$, RPAT samples had RINs of $7.1 \pm 0.4$, VAT sample RINs was $5.5 \pm 0.4$ and neck SAT samples had RINs of $6.8 \pm 0.2$.

\section{Gene expression}

The gene expression analysis was performed as described in (Rødgaard et al. 2012). Briefly, cDNA syntheses of the samples were performed in duplicate (technical replicates) using $500 \mathrm{ng}$ of total RNA. This was reverse transcribed using the QuantiTECT Reverse Transcription kit (Qiagen), according to the manufacturer's instructions. Pre-amplification was performed using TaqMan PreAmp Master Mix (Applied Biosystems).

Primers were designed as described previously in the study by Skovgaard et al. (2009). Gene symbols, primer sequences and amplicon lengths are shown in Table S1. Primer amplification efficiencies, dynamic ranges, specificity and sequences were analyzed as described in the study by Rødgaard et al. (2012).

Quantitative PCR was performed in 48.48 Dynamic Array Integrated Fluidic Circuits (Fluidigm), combining 48 samples with 48 primer sets in the BioMark Real-Time PCR instrument (Fluidigm). Expression data were acquired using the Fluidigm REAL-TIME PCR ANALYSIS software 3.0.2 (Fluidigm) and exported to GENEX5 (MultiD, Göteborg, Sweden).

\section{Serum protein quantification}

Serum protein concentrations were determined using sandwich enzyme-linked immunosorbent assays (ELISAs). Commercially available ELISA assays were used for interleukin 6 (IL-6) (Porcine IL-6 Duoset kit, R\&D Systems, Inc.), tumor necrosis factor- $\alpha$ (TNF- $\alpha$ ) (Swine TNF- $\alpha$ CytoSet $^{\mathrm{TM}}$, with Antibody Pair Buffer Kit; Invitrogen) and serum amyloid A (SAA) (Phase SAA assay, Tridelta Development Ltd.) according to manufacturers' protocol. C-reactive protein (CRP) and haptoglobin (HP) were analyzed with in-house assays as described in Rødgaard et al. (2012). TMB-plus (Kem-En-Tec) was used for developing the ELISA- 
plates, according to the manufacturer's instructions. Plates were then read using an automatic plate reader (Thermo Multiskan Ex spectrophotometer, Thermo Scientific). ASCENT software v. 2.6 (Thermo Scientific) was used to calculate sample values calibrating each plate with several dilutions of a standard solution of known concentration of the protein in question.

\section{Data analysis and statistics}

For qPCR data pre-processing, normalization and relative quantification GENEX5 was used. Out of a panel of five putative reference genes, the most stably expressed were selected for normalization (for liver: GAPDH, B2M, RPL13A and $A C T B$; for the adipose tissues all five were chosen: HPRT1, GAPDH, B2M, RPL13A and ACTB) using GeNorm (Vandesompele et al. 2002). The geometric mean of these was used for normalization of each tissue individually in GENEX5. To calculate relative expression levels, the $\mathrm{Cq}$ values were calculated relative to the least expressed sample for each primer set. The data from qPCR and ELISAs were $\log 2$ transformed to approach normal distribution prior to two-tailed, unpaired $t$-test, one-way ANOVA with TukeyKramer post-test and/or $F$-test, and $P \leq 0.05$ was considered significant. Statistics were performed in GENEX5 or PRISM5 (GraphPad Software, Inc.). Data are expressed as the mean \pm standard error of the mean (SEM).

\section{Results}

The mean daily feed intake of the pigs in the obese group having free access to food was around $1.5 \mathrm{~kg}$. At euthanasia, the body weight of the pigs of the obese group $(92.6 \pm 5.2 \mathrm{~kg}$; mean and SEM respectively) was significantly $(P<0.0001)$ higher than was the body weight in the lean group $(50.3 \pm 1.6 \mathrm{~kg})$.

\section{Gene expression}

The expression profiles of 39 genes related to innate immunity were analyzed as were five putative reference genes. In the pre-processing of data, several genes were removed from further analysis due to low efficiencies or due to high variation between the technical replicates (as described in Rødgaard et al. (2012): FGG, IL1B, IL8(a), IL8(b), AOAH, IL1O(b), ORM1(a), ORM1(b), SFTPA1 and IFNG were removed from the fatty tissue data and $I L 1 B$, AOAH, SFTPA1, ORM1(a), ORM1(b), ADIPOQ, LBP and IL8

(a) from the liver tissue data.

Genes differentially expressed between lean and obese pigs at significance level $P=0.05$ and with a change of at least \pm 1.5 -fold are depicted for all four tissues in Fig. 1. Overall, obesity resulted in a significantly changed expression of 12 genes in VAT (nine of which were downregulated in obese animals), 11 genes in RPAT (of which seven were up-regulated in the obese group) and eight genes in neck SAT (three of these genes being downregulated). The adiponectin gene was down-regulated in both VAT and RPAT of the obese pigs. The genes significantly correlated with obesity in the liver were mostly down-regulated (six of nine).

Three genes were significantly differentially regulated between the lean and obese minipigs in all three adipose tissues, namely the chemokine (C-C motif) ligand 3-like 1 (CCL3L1), interleukin 1 receptor antagonist (IL1RN) and CD200 molecule (CD200), with CCL3L1 and IL1RN being up-regulated and CD2OO being down-regulated in all three adipose tissue types of obese pigs. In the liver, none of these three genes changed expression in obese animals.

The pattern of tissue-specific adipose tissue gene expression also may be compared between lean and obese pigs (see Table 1). For both groups of pigs, approximately half of the investigated genes showed different expression levels in the three types of adipose tissues. In the lean group, all of these genes were expressed at higher levels in RPAT and VAT than in neck SAT (19 genes), whereas in the obese pigs, the number of genes being more highly expressed in RPAT and VAT compared to neck SAT was lower (10 genes). Specifically, in the obese group, more genes encoding for acute phase proteins (APPs) were significantly differentially expressed among adipose tissues, with six genes (C3, CRP, HP, ITIH4(a), ITIH4(b), LBP and SAA) compared with two genes ( $C 3$ and $C R P)$ in the lean minipigs; in contrast, only two genes encoding for cytokines (TGFB1 and IL1RN) and none encoding for chemokines were significantly differentially expressed in adipose tissues in the obese animals, compared with four genes encoding for cytokines (IL1O, IL18, TGFB1 and TNFAIP3) and three encoding chemokines (CCL3L1 and CXCL1O) in the adipose tissues of lean minipigs.

\section{Serum protein concentrations}

The serum concentrations of HP, CRP and SAA are depicted in Fig. S1. No significant differences in HP, CRP or SAA serum concentrations were found between the lean and obese Göttingen minipigs. The serum levels of TNF- $\alpha$ and IL6 were below the detection limit (except in one lean minipig in the IL-6 ELISA) and are therefore not depicted. None of the transcripts coding for these proteins were significantly differentially regulated in the liver of obese compared with lean pigs; however, in the VAT, the APPs encoded by HP and $S A A$ were significantly differentially down-regulated in the obese minipigs compared with the lean minipigs and IL6 was up-regulated in both the RPAT and the VAT in the obese minipigs compared with the lean (Fig. 1). 

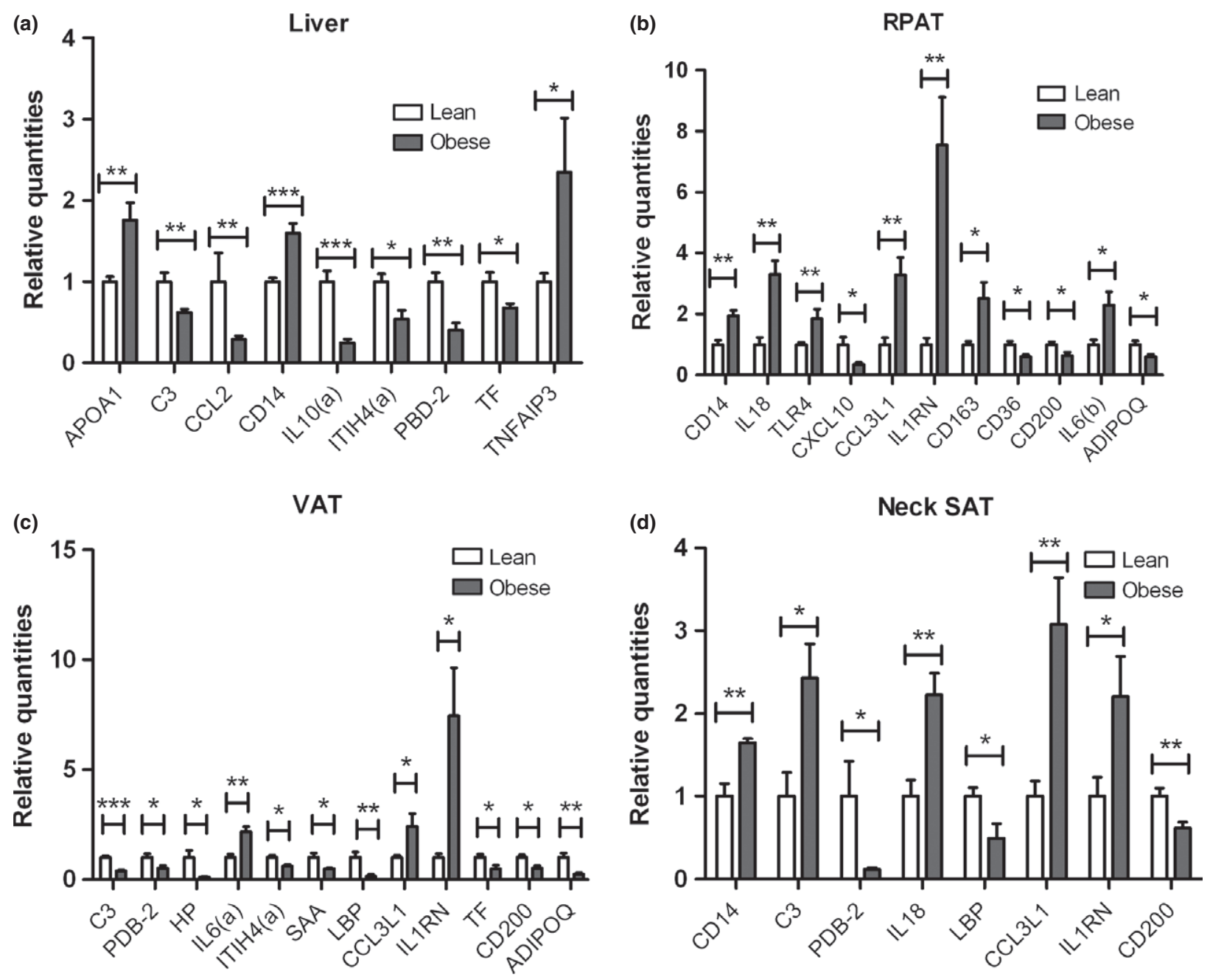

Figure 1 Significantly differentially expressed genes in lean compared with obese Göttingen minipigs. Only genes with a fold change equal to or greater than \pm 1.5 [compared with gene expression in lean pigs (set to 1 for each gene)] in liver (a), retroperitoneal adipose tissue (RPAT) (b), visceral adipose tissue (VAT) (c) and neck subcutaneous adipose tissue (SAT) (d) as determined by qPCR are included. In the VAT, both primers for IL6 showed similar patterns and significance (**); therefore, IL6(b) was removed from the graph. For all tissues, lean $n=7$ and obese $n=7$. Error bars depict standard error of the mean. $* P<0.05, * * P<0.01, * * * P<0.001$

\section{Discussion}

Obesity-related changes in the expression of innate immune system-related genes and possible similarities to human obesity of obese Göttingen minipigs have to our knowledge not been thoroughly described before. Here, we studied Göttingen minipigs that were either allowed free access (obese group) or allowed restricted access (lean group) to normal pig chow. Thus, the effects of food intake and obesity cannot be differentiated in this model as they are highly correlated.

The obese Göttingen minipigs had significantly higher bodyweights than did the lean pigs, and we find clear differences in the gene regulation in the obese minipigs compared with lean, including for genes encoding APP and cytokines in adipose tissues (Fig. 1). The expression of a number of other relevant obesity-related genes in these pigs will be published elsewhere (S. Cirera et al. 2013).

Interestingly, three genes were significantly differentially regulated in all three adipose tissues of the obese pigs as compared with the lean pigs, namely CCL3L1, IL1RN and CD2OO (Fig. 1). CCL3L1 is up-regulated in the obese minipigs and encodes a chemokine, also known as macrophage inflammatory protein $1-\alpha$ (MIP- $1 \alpha$ ), that is involved in acute inflammation by recruiting and activating polymorphonuclear leukocytes (Wolpe et al. 1989). Previous work in mice has established that this gene is up-regulated in the epididymal white adipose tissue of obese mice and may play a role in the development of type II diabetes (Xu et al. 2003). To our knowledge, the gene expression of CCL3L1 has not yet been investigated in human adipose tissues; however, the serum protein concentration is similar in lean and obese humans (Kim et al. 2006). The 


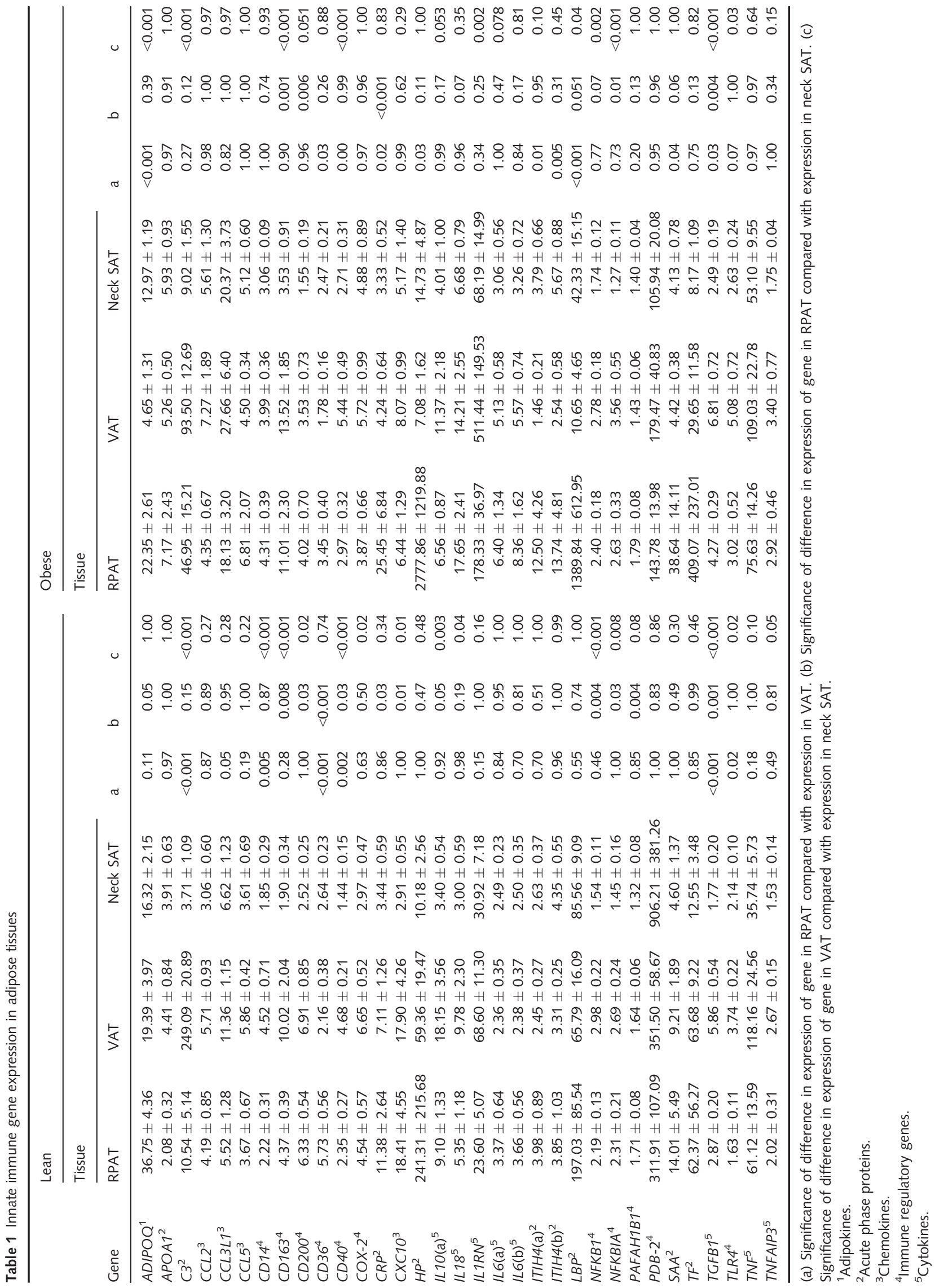


up-regulation of this pro-inflammatory gene (CCL3L1) is accompanied by the down-regulation of the anti-inflammatory gene CD200. CD200 is a receptor on the surface of macrophages, for example, and helps down-regulate myeloid cell activity (Hoek et al. 2000; Wright et al. 2000). The combined effect of these changes in the adipose tissues of the obese minipigs compared with the lean would be proinflammatory. However, the most up-regulated gene in VAT and RPAT of obese pigs is IL1RN. The product of IL1RN is the interleukin 1 receptor antagonist (IL1ra), a naturally occurring inhibitor of the pro-inflammatory proteins IL- $1 \alpha$ and $-\beta$ (Seckinger et al. 1987). White adipose tissue has been found to be a major source of IL1ra in both mice and men (Juge-Aubry et al. 2003), and IL1ra may play a role in acquired resistance to leptin in obesity in rats and humans (Luheshi et al. 1999; Meier et al. 2002). IL1RN is significantly up-regulated in the VAT compared with neck SAT in the obese pigs $(P=0.002)$ (Table 1$)$, which could counteract the pro-inflammatory effects of the down-regulated CD2OO and the up-regulated CCL3L1. This might explain the absence of changes in serum cytokine and APP concentrations in the obese Göttingen minipigs studied here. Interestingly, IL6 is up-regulated in the RPAT and VAT in the obese minipigs, and previous reports suggest that IL6 suppresses inflammation via induction of IL1ra synthesis in humans (Tilg et al. 1994). This is supported by the findings that the up-regulation of ILIRN expression in the RPAT and VAT, where IL6 is up-regulated, is much higher than in the neck SAT (7.5 times higher in the RPAT and VAT of the obese pigs and only two times higher in the neck SAT of the obese minipigs compared to lean) (Fig. 1b-d).

A correlation between increased adipose tissue expression and elevated serum levels of various innate immune factors in obesity has been established in humans for a number of proteins, including IL-6 (Fontana et al. 2007), HP (Chiellini et al. 2004) and SAA (Poitou et al. 2006). However, such correlations were not found in the Göttingen minipigs investigated in the present study. Of the three genes mentioned, adipose tissue expression of only IL6 was elevated, in both the RPAT and the VAT (Fig. 1); however, no increases in serum concentration of IL- 6 were observed in the Göttingen minipigs. The expression of both $S A A$ and HP was down-regulated in the VAT, and no changes in serum concentrations of these proteins were detected either. This contrasts with the serum protein changes observed in the human response to obesity, in which CRP, SAA and HP serum concentrations are elevated (van Dielen et al. 2001; Esposito et al. 2003; Chiellini et al. 2004). A reason for this could be that the fold changes of the genes significantly correlated with obesity in the liver are very small (Fig. 1a) and perhaps too small for the changes to be translated into elevated serum protein concentration. Another reason why the Göttingen minipigs in this study do not show a serum response to obesity similar to humans could be that their diet contained no excess fat or sugar, and it is possible that a standard diet does not induce a systemic or local low-grade inflammation. However, the pigs had been obese for a prolonged period of time, as they were euthanized at 4147 months, which mimics the human chronic obesity state.

In summary, a wide range of innate immunology-related genes are significantly differentially regulated in the obese Göttingen minipigs compared with the lean pigs. However, in contrast to human obesity, this does not translate into changes in serum concentrations of cytokines and APPs in the obese pigs compared with the lean. This should be taken into account when selecting biomarkers for obesity in Göttingen minipigs.

\section{Acknowledgements}

Karin Tarp Wendt is acknowledged for exceptional help with qPCR. The lean Göttingen minipigs were funded by the SHARE Foundation and Danielsen's Foundation.

\section{References}

Bastard J.-P., Maachi M., Lagathu C., Kim M.J., Caron M., Vidal H., Capeau J. \& Feve B. (2006) Recent advances in the relationship between obesity, inflammation, and insulin resistance. European Cytokine Network 17, 4-12.

Chiellini C., Santini F., Marsili A. et al. (2004) Serum haptoglobin: a novel marker of adiposity in humans. Journal of Clinical Endocrinology and Metabolism 89, 2678-83.

Cirera S, Jensen M.S., Elbrønd V.S. et al. (2013) Expression studies of six human obesity-related genes in seven tissues from divergent pig breeds. Animal Genetics, DOI: 10.1111/age.12082.

van Dielen F.M.H., van't Veer C., Schols A.M., Soeters P.B., Buurman W.A. \& Greve J.W.M. (2001) Increased leptin concentrations correlate with increased concentrations of inflammatory markers in morbidly obese individuals. International Journal of Obesity 25, 1759-66.

Esposito K., Pontillo A., Giugliano F., Giugliano G., Marfella R., Nicoletti G. \& Giugliano D. (2003) Association of low interleukin10 levels with the metabolic syndrome in obese women. Journal of Clinical Endocrinology and Metabolism 88, 1055-8.

Federico A., D’Aiuto E., Borriello F., Barra G., Gravina A.G., Romano M. \& De Palma R. (2010) Fat: a matter of disturbance for the immune system. World Journal of Gastroenterology 16, 4762-72.

Fontana L., Eagon J.C., Trujillo M.E., Scherer P.E. \& Klein S. (2007) Visceral fat adipokine secretion is associated with systemic inflammation in obese humans. Diabetes 56, 1010-3.

Gil A., Aguilera C.M., Gil-Campos M. \& Cañete R. (2007) Altered signalling and gene expression associated with the immune system and the inflammatory response in obesity. British Journal of Nutrition 98, S121-6.

Hoek R.M., Ruuls S.R., Murphy C.A. et al. (2000) Down-regulation of the macrophage lineage through interaction with OX2 (CD200). Science 290, 1768-71.

Johansen T., Hansen H.S., Richelsen B. \& Malmlöf K. (2001) The obese Göttingen minipig as a model of the metabolic syndrome: dietary effects on obesity, insulin sensitivity, and growth hormone profile. Comparative Medicine 51, 150-5. 
Juge-Aubry C.E., Somm E., Giusti V., Pernin A., Chicheportiche R., Verdumo C., Rohner-Jeanrenaud F., Burger D., Dayer J.M. \& Meier C.A. (2003) Adipose tissue is a major source of interleukin1 receptor antagonist. Diabetes 52, 1104-10.

Kim C.S., Park H.S., Kawada T., Kim J.H., Lim D., Hubbard N.E., Kwon B.S., Erickson K.L. \& Yu R. (2006) Circulating levels of MCP-1 and IL-8 are elevated in human obese subjects and associated with obesity-related parameters. International Journal of Obesity 30, 1347-55.

Larsen M.O., Rolin B., Wilken M., Carr R.D. \& Svendsen O. (2002) High-fat high-energy feeding impairs fasting glucose and increases fasting insulin levels in the Göttingen minipig. Annals of the New York Academy of Sciences 967, 414-23.

Larsen M.O., Rolin B., Raun K., Knudsen L.B., Gotfredsen C.F. \& Bock T. (2007) Evaluation of $\beta$-cell mass and function in the Göttingen minipig. Diabetes, Obesity and Metabolism 9, 170-9.

Litten-Brown J.C., Corson A.M. \& Clarke L. (2010) Porcine models for the metabolic syndrome, digestive and bone disorders: a general overview. Animal 4, 899-920.

Luheshi G.N., Gardner J.D., Rushforth D.A., Loudon A.S. \& Rothwell N.J. (1999) Leptin actions on food intake and body temperature are mediated by $\mathrm{IL}-1$. Proceedings of the National Academy of Sciences of the United States of America 96, 704752.

Meier C.A., Bobbioni E., Gabay C., Assimacopoulos-Jeannet F., Golay A. \& Dayer J.M. (2002) IL-1 receptor antagonist serum levels are increased in human obesity: a possible link to the resistance to leptin? Journal of Clinical Endocrinology and Metabolism 87, 1184-8.

Nguyen T. \& Lau D. (2012) The obesity epidemic and its impact on hypertension. Canadian Journal of Cardiology 28, 326-33.

Poitou C., Coussieu C., Rouault C. et al. (2006) Serum amyloid A: a marker of adiposity-induced low-grade inflammation but not of metabolic status. Obesity 14, 309-18.

Rødgaard T., Skovgaard K., Stagsted J. \& Heegaard P.M.H. (2012) Expression of innate immune response genes in liver and three types of adipose tissue in cloned pigs. Cellular Reprogramming 14 407-17.
Seckinger P., Lowenthal J.W., Williamson K., Dayer J.M. \& MacDonald H.R. (1987) A urine inhibitor of interleukin 1 activity that blocks ligand binding. Journal of Immunology 139 , 1546-9.

Skovgaard K., Mortensen S., Boye M., Poulsen K., Campbell F., Eckersall P. \& Heegaard P. (2009) Rapid and widely disseminated acute phase protein response after experimental bacterial infection of pigs. Veterinary Research 40, 23.

Tilg H., Trehu E., Atkins M.B., Dinarello C.A. \& Mier J.W. (1994) Interleukin-6 (IL-6) as an anti-inflammatory cytokine: induction of circulating IL-1 receptor antagonist and soluble tumor necrosis factor receptor p55. Blood 83, 113-8.

Vandesompele J., De Preter K., Pattyn F., Poppe B., Van Roy N., De Paepe A. \& Speleman F. (2002) Accurate normalization of realtime quantitative RT-PCR data by geometric averaging of multiple internal control genes. Genome Biology 3, research0034.1-11.

Wolpe S.D., Davatelis G., Sherry B., Beutler B., Hesse D.G., Nguyen H.T., Moldawer L.L., Nathan C.F., Lowry S.F. \& Cerami A. (1989) Macrophages secrete a novel heparin-binding protein with inflammatory and neutrophil chemokinetic properties. Journal of Experimental Medicine 167, 570-81.

Wright G.J., Puklavec M.J., Willis A.C., Hoek R.M., Sedgwick J.D., Brown M.H. \& Barclay A.N. (2000) Lymphoid/neuronal cell surface OX2 glycoprotein recognizes a novel receptor on macrophages implicated in the control of their function. Immunity 13, 233-42.

Xu H., Barnes G.T., Yang Q. et al. (2003) Chronic inflammation in fat plays a crucial role in the development of obesity-related insulin resistance. Journal of Clinical Investigation 112, 1821-30.

\section{Supporting information}

Additional supporting information may be found in the online version of this article.

Figure S1 Serum protein concentrations.

Table S1 Primers used for high-throughput qPCR. 